\title{
A Child with Unusual Liver Lesions in a Case of Neuroblastoma
}

A 21-month-old female initially presented to us with a 2-week history of ataxia, loss of consciousness, bruises on the forehead, nystagmus, tremor, and mydriasis with morning irritability. The findings of magnetic resonance imaging (MRI) of the abdomen were consistent with a neuroblastoma [Figure 1]. At the time of diagnosis, the tumor was localized and showed no evidence of local or regional invasion, as defined by the image-defined risk factors.

On surgery, the tumor was completely excised, with clear margins representing Stage 1 of the International Neuroblastoma Staging System. Histopathology was favorable with differentiating subtype and low Mitosis-Karyorrhexis index. Post surgery, MRI of the abdomen demonstrated no residual soft tissue. This was followed by intravenous immunoglobulin, prednisone, and cyclophosphamide according to standard regimen to treat the opsoclonus myoclonus ataxia syndrome. A stem cell collection was performed for autologous stem cell procedure.
At 3-month follow-up, no tumor recurrence was seen at the surgical bed. However, a single, small lesion with equivocal enhancement was discovered within the liver in segment $6 / 5$, which was thought to be metastases versus hemangioma. Multiple follow-up MRI at 3-month interval showed gradually increasing number and size of lesions in the liver with avid enhancement but no specific washout characteristics. The biopsy of this liver lesion was attempted on two separate occasions, without a definitive diagnosis. The lesions stabilized 2 years postoperatively [Figure 2]. Still, as the possibility of metastases was thought to be highly likely, the tumor board decided to pursue open biopsy for diagnosis. The pathology on the peripheral wedge biopsy of liver surprisingly again showed no evidence of neoplasia [Figure 3]. What could be the cause of such an appearance?

\section{Answer}

Neuroblastoma is a common neoplasm in the pediatric age group, usually presenting between 1 and 5 years of age. Metastases are frequent in bones and lymph nodes. Rarely,
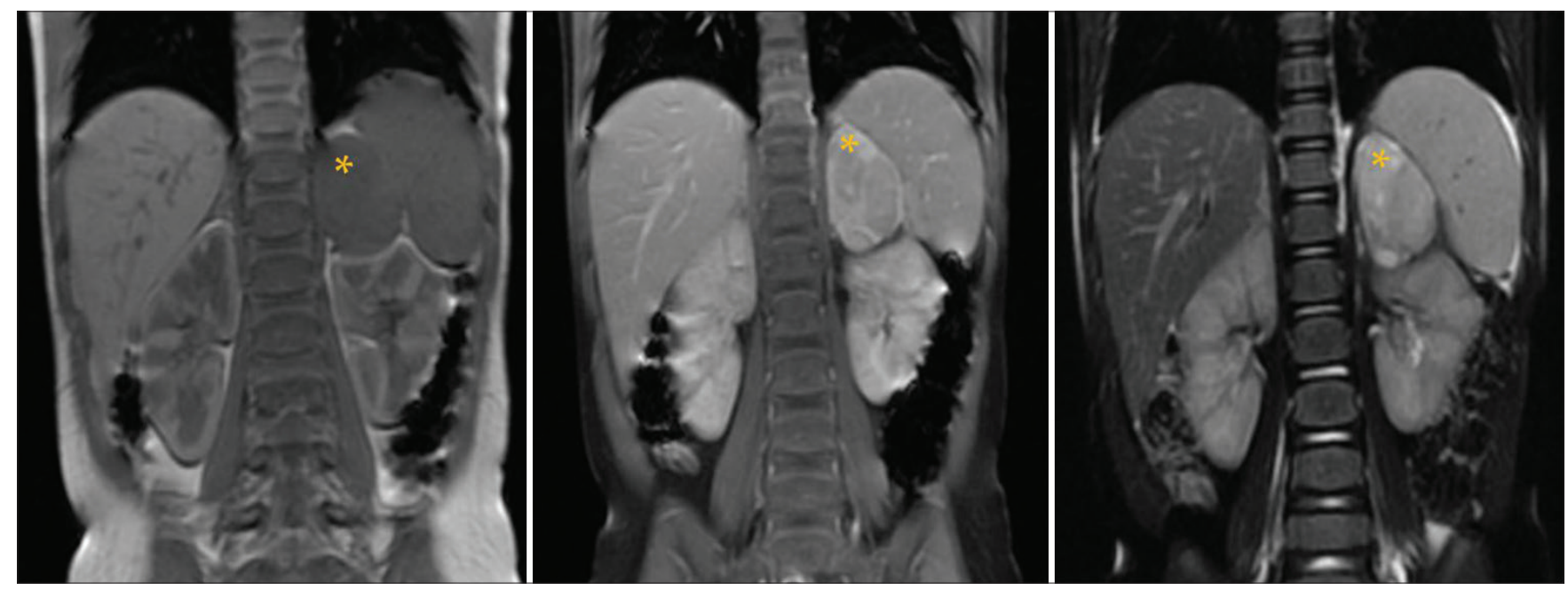

Figure 1: Coronal T1, T1c+, and T2 images: A large left suprarenal lobulated lesion appearing hypointense on T1-weighted image with heterogeneous postcontrast enhancement. No signal voids are seen on T2-weighted image. The lesion does not cross the midline. Mass effect is seen on the adjacent spleen/kidney without invasion. There was no extension into the adjacent neural foramina. Note that the visualized liver appears normal 

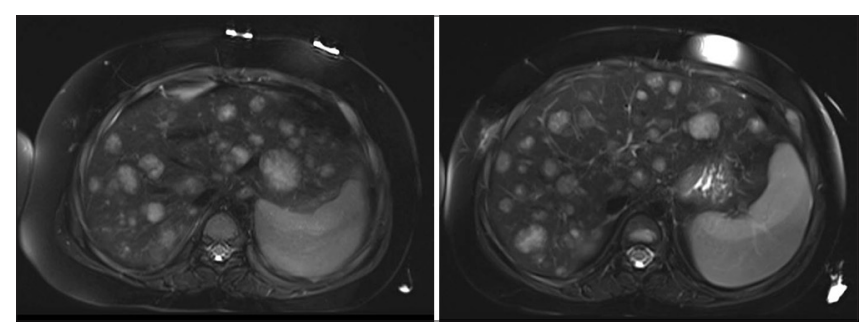

Figure 2: Axial T2 images: Stable large liver lesions 2 years after the surgery. These lesions were found to be in keeping with extramedullary hematopoiesis

they may also be seen within the visceral organs and soft tissues.

Extramedullary hematopoiesis (EMH) is the formation of normal blood cells outside of the bone marrow, which is seen as a sequela of inadequate hematopoiesis. ${ }^{[1]}$ The actual pathogenesis of EMH in patients with solid tumors remains unknown. Granulocyte colony-stimulating factor (G-CSF) is an important inducing growth factor which stimulates the bone marrow to increase the production and release of granulocytes into the blood. ${ }^{[2]} \mathrm{G}-\mathrm{CSF}$ in turn may be an inducing factor of EMH. Our patient had received G-CSF as a stem cell rescue to ameliorate marrow suppression from chemotherapy.

A recent review described the presence of EMH in a spectrum of malignant solid tumors. They also describe liver as the most common visceral organ for EMH followed by kidney and paraspinal region..$^{[2]}$ However, to the best of our knowledge, the association of neuroblastoma and EMH in liver has not been reported in literature.

To conclude, in the setting of an existing malignancy, specifically neuroblastoma, EMH may pose as metastatic deposits signifying the progression of disease. In addition, childhood cancer survivors are at risk of developing pseudometastatic mass lesions, which are often multiple and develop years after completing chemotherapy. Due to prognostic implications, it is essential to confirm these lesions as they are nonaggressive with a benign course.

\section{Declaration of patient consent}

The authors certify that they have obtained all appropriate patient consent forms. In the form the patient(s) has/have given his/her/their consent for his/her/their images and other clinical information to be reported in the journal. The patients understand that their names and initials will not be published and due efforts will be made to conceal their identity, but anonymity cannot be guaranteed.

\section{Financial support and sponsorship}

Nil.

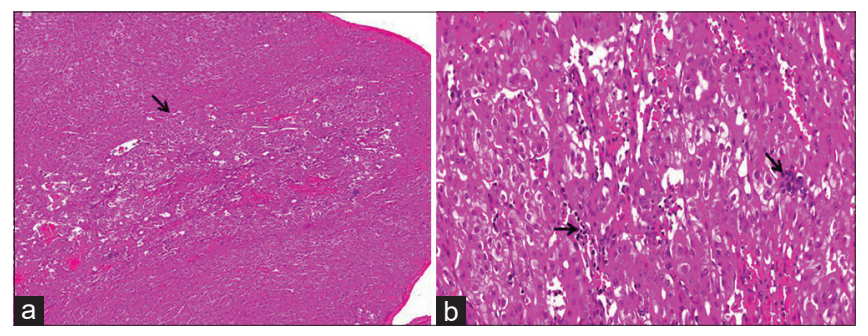

Figure 3: Postbiopsy: (a) Low-power liver parenchyma showing a vague nodule with the arrow marking the nodule of extramedullary hematopoiesis. Contrast between dilated sinusoids in the nodule and unremarkable surrounding liver parenchyma (hematoxylin phloxine saffron stain, $\times 40$ ), (b) medium-power views of the liver parenchyma showing dilated sinusoids, extramedullary hematopoiesis, and sinusoidal capillarization (hematoxylin phloxine saffron stain, $\times 200$ )

\section{Conflicts of interest}

There are no conflicts of interest.

Abhijeet Taori ${ }^{1}$, Divya Malpani ${ }^{2}$

${ }^{\prime}$ Department of Radiodiagnosis, Suyash Hospital, Indore, Madhya Pradesh, India, '2Department of Radiodiagnosis, Apollo Hospital, Indore, Madhya Pradesh, India

Address for correspondence: Dr. Abhijeet Taori, Department of Radiodiagnosis, Suyash Hospital, Indore, Madhya Pradesh, India. E-mail: abhijittaori@gmail.com

Submitted: 28 -Apr-2020

Revised: 06-Jul-2020 Accepted: 06-Aug-2020 Published: 29-Aug-2020

\section{References}

1. Kim CH. Homeostatic and pathogenic extramedullary hematopoiesis. J Blood Med 2010;1:13-9.

2. Bao Y, Liu Z, Guo M, Li B, Sun X, Wang L. Extramedullary hematopoiesis secondary to malignant solid tumors: A case report and literature review. Cancer Manag Res 2018;10:1461-70.

This is an open access journal, and articles are distributed under the terms of the Creative Commons Attribution-NonCommercial-ShareAlike 4.0 License, which allows others to remix, tweak, and build upon the work non-commercially, as long as appropriate credit is given and the new creations are licensed under the identical terms.

\begin{tabular}{|l|l|}
\hline \multicolumn{2}{|c|}{ Access this article online } \\
\hline Quick Response Code: & Website: \\
& www.ijmpo.org \\
\cline { 2 - 2 } & DOI: \\
\hline
\end{tabular}

How to cite this article: Taori A, Malpani D. A child with unusual liver lesions in a case of neuroblastoma. Indian J Med Paediatr Oncol 2020;41:565-6. 